\title{
Platform Angle Signal Blind Source Separation Algorithm Based on Wavelet -EEMD Method
}

\author{
Yuegang Wang, ${ }^{1, \mathrm{a}}$, Wuxing Huang, ${ }^{1, \mathrm{~b}}$, Zhibin Guo ${ }^{1, \mathrm{c}}$, Qiangqiang \\ Ren $^{1, \mathrm{~d}}$, Le Wang,e \\ ${ }^{1} \mathrm{Xi}$ 'an Research Inst. of Hi-Tech, Hongqing Town,Xi'an, P.R.China \\ aemail:wangyueg@163.com, ${ }^{b}$ email:439805636@qq.com,cemail:gzbcapt \\ ain@163.com, ${ }^{d}$ email:1101112462@qq.com, \\ eemail:15667081181@qq.com
}

\begin{abstract}
To solve the problem that self-alignment accuracy of platform inertial navigation system will be affected a lot under significant interference, an adaptive blind source separation algorithm based on wavelet - EEMD method is proposed. This method use wavelet decomposition to remove the high frequency signal, and then the EEMD can be used to decompose the target signal. While using wavelet decomposition, an algorithm that using volatility to determine the decomposition layers is proposed, and this method increased the adaptability of wavelet decomposition algorithm. Finally, experiment data validation and some compare are carried on to confirm the proposed method, and the results show that the method has a great advantage, and it can extract the target signals from the disturbed data under significantly interference and greatly improve the alignment accuracy of platform inertial navigation system.
\end{abstract}

Keywords: Platform inertial navigation system; Wavelet decomposition; Ensemble experience mode decomposition(EEMD)

\section{Introduction}

Platform inertial navigation system is often interfered by complex surroundings during actual alignment process, to solve this problem, there were a lot of researches done by scholars, but the problem that the alignment accuracy is influenced under the circumstance of substantial rocking motion of the base was not well solved.

Consider INS signal interference at the perspective of signal decomposition, the ideal frame angle sensor output signal can be got by signal decomposition. Traditional methods, such as Wiener filtering, Kalman filtering, etc., need to pre-determine the filter system or a related model parameters and noise filtering effects differ from the forms of noise, so traditional methods are not suitable for blind signal separation which was lack of prior knowledge of the interfering signal[1]. Ideal frame angle sensor output signal is low-frequency and has a monotonic trend, therefore, many scholars tried Fourier filtering algorithm and 
wavelet filtering algorithm to remove high frequency noise, but the problem of glitch failed to be solved;

EEMD (ensemble empirical mode decomposition) [2], based on the signal characteristic scales, decomposes signal into a number of different scales of intrinsic characteristics intrinsic functions (IMF, Intrinsic Mode Function). Not only can this method reflect distribution of signal energy on different scales of time and space exactly, but also overcome the modal aliasing of EMD algorithm. The trend term of decomposition results is the ideal frame angle sensor output signal. However, for the disturb output under the circumstance of substantial rocking motion of the base, the trend term inevitably have trend interference caused by significant interference, which will invoke large errors.

Based on the characteristics that the signal is low-frequency and has a monotonic trend, the method of combining the wavelet decomposition and EEMD to decompose signal is proposed. Firstly, the signal is decomposed by wavelet decomposition, and the high frequency parts are removed as much as possible in the context of keeping the target signal integrated. Then, the resulting low-frequency signal is decomposed by EEMD, the trend term of the results is ideal frame angle sensor output signal. The experimental data validation shows that this method can overcome the influence of various interfering signals and have good effect on significant interference.

\section{Wavelet decomposition}

On account of the low-frequency characteristics of the target signal, before using EEMD, wavelet decomposition can be used to filter high frequency noise part to improve the accuracy of EEMD decomposition. Multi-resolution wavelet decomposition [3] is a binary recursive decomposition model, which can be described as follows: If the total band of original signal s(n) is defined as $v_{0}(0 \sim \pi)$, after the first level decomposition, $v_{0}$ was divided into low frequency part $v_{1}(0 \sim \pi / 2)$ and high frequency part $w_{1}(\pi / 2 \sim \pi)$; After the second level decomposition, $v_{1}$ are separated into low frequency part $v_{2}(0 \sim \pi / 4)$ and high frequency part $w_{2}(\pi / 4 \sim \pi / 2)$.By that analogy, after n-level decomposition, the low-frequency part of the signal is $v_{n}\left(0 \sim \pi / 2^{n}\right)$.

The most important step of wavelet decomposition is the selection of wavelet bases and decomposition level. The selection of Wavelet bases needs to take the characteristics of signal into consideration, and try to remove high frequency noise as much as possible in the decomposition, while keeping the original characteristics of signal [4]. Since db5 wavelets is Regularity and has good locality in frequency, the paper selects db5 wavelets.

The interference signal is uncertain, and the distribution of interference signal will influence the selection of wavelet decomposition level, wavelet decomposition levels are not unique. If interference signal only exists high 
frequency engine vibration part, the decomposition level is small; while low frequency significant interference exists, decomposition level should be large enough. The wavelet decomposition itself is not adaptive, its application is limited. To solve this problem the paper proposed volatility method to select signal decomposition level.

Since the larger the amplitude of interference signal is, the bigger the influence on target signal will be, and volatility will be more intense, wavelet decomposition level should be greater too. decomposition level can be selected according to the volatility. Volatility is used to describe the fluctuation degree of interfered signal, it can be calculated as follows:

$$
b=\frac{l_{\max }^{\prime}-l_{\min }^{\prime}}{l_{\max }-l_{\min }}
$$

$l$ is the amplitude of the signal, $l_{\max }-l_{\min }$ is the amplitude interval length, $l^{\prime}$ is amplitude of the subtraction of ideal signal and mixed signal, $l_{\max }^{\prime}-l_{\min }^{\prime}$ is the maximum fluctuation value of mixed signal. Ideal signal is unknown, so the least-squares curve fitting of mixed signal can be used as the reference to seek maximum fluctuation value.

After several experiments, the corresponding rules between volatility b and decomposition level $\mathrm{n}$ is shown in Tab. 1.

Tab. 1 Corresponding rules between volatility $\mathrm{b}$ and decomposition level $\mathrm{n}$

\begin{tabular}{|c|c|c|c|c|c|c|c|c|c|}
\hline $\mathrm{b}$ & $<0.03$ & $<0.05$ & $<0.07$ & $<0.1$ & $<0.13$ & $<0.18$ & $<0.24$ & $<0.35$ & $>0.35$ \\
\hline $\mathrm{n}$ & 0 & 2 & 3 & 4 & 5 & 6 & 7 & 8 & 9 \\
\hline
\end{tabular}

According to the rules, corresponding decomposition level $\mathrm{n}$ for each volatility b can be gotten, thus it's a good solution to wavelet's lacking of adaptability.

\section{Ensemble empirical mode decomposition (EEMD)}

The main principle of empirical Mode Decomposition (EMD)[5] is to decompose signal into several intrinsic mode functions (IMFs), and each IMF has real physical meaning and high credibility, so EMD is suitable for extracting framework angle sensor signal. However, EMD method has aliasing modes, which have impact on the separation results. To overcome EMD modal aliasing, Wu et al [5] proposed ensemble empirical mode decomposition (EEMD) through statistical characteristics of white noise signal, adding white noise into the original signal before EMD decomposition, the final IMF is the average of multiple decomposition results.

EEMD method specific steps [6] can be described as follows:

(1) Add random Gaussian white noise $u_{i}(t)$ In a mixed signal $s(t)$ repeatedly, ie.

$$
s_{i}(t)=s(t)+u_{i}(t)
$$


Where: $s_{i}(t)$ is the $i$ th add white Gaussian noise signals, $u_{i}(t)$ is the random Gaussian white noise.

(2) Decompose $s_{i}(t)$ by EMD, IMF component denoted as $c_{i j}(t)$, margin denoted as $r_{i}(t)$. Here, $c_{i j}(t)$ is the number $j$ IMF components when the $i$ th adding white noise and decomposition.

(3) Repeat steps (1)-(2) N times. According to the principle that statistic of uncorrelated random sequence is zero, we can eliminate the influence of repeatedly adding white noise by averaging all corresponding IMFs. The resulting IMF can be described as follows:

$$
c_{j}(t)=\frac{1}{N} \sum_{i=1}^{N} c_{i j}
$$

Where, $c_{j}(t)$ is the number $j$ IMF components decomposed by EEMD. The residue component can be expressed as:

$$
r(t)=\frac{1}{N} r_{i}(t)
$$

Finally, results of EEMD decomposition can be described as:

$$
s(t)=\sum_{j} c_{j}(t)+r(t)
$$

The residual signal, which is the ideal target signal according to the previous analysis, represents the average trend. To make the decomposition results more accurate, this paper set Gaussian white noise SNR to 0.2, set EMD decomposition level $\mathrm{N}$ to 100.

\section{Extraction of platform frame angle signal}

Since low volatility signals can be decomposed easily, here we mainly study the larger interference signals collected during the experiment, as shown in Fig. 1. 


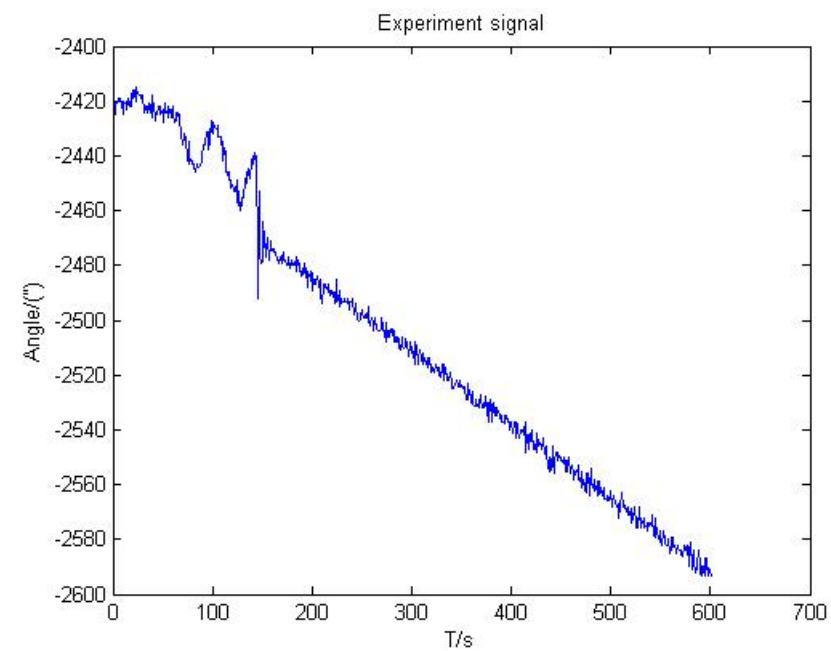

Fig. 1 Larger magnitude interference experimental data

Fig. 1 shows that signal was interfered by the large interference during time $0-150$, resulting in a large deviation. General filtering method and signal decomposition method are difficult to eliminate such a significant interference, separation results are greatly influenced by substantial interference. Here is residual analysis of three filtering methods.

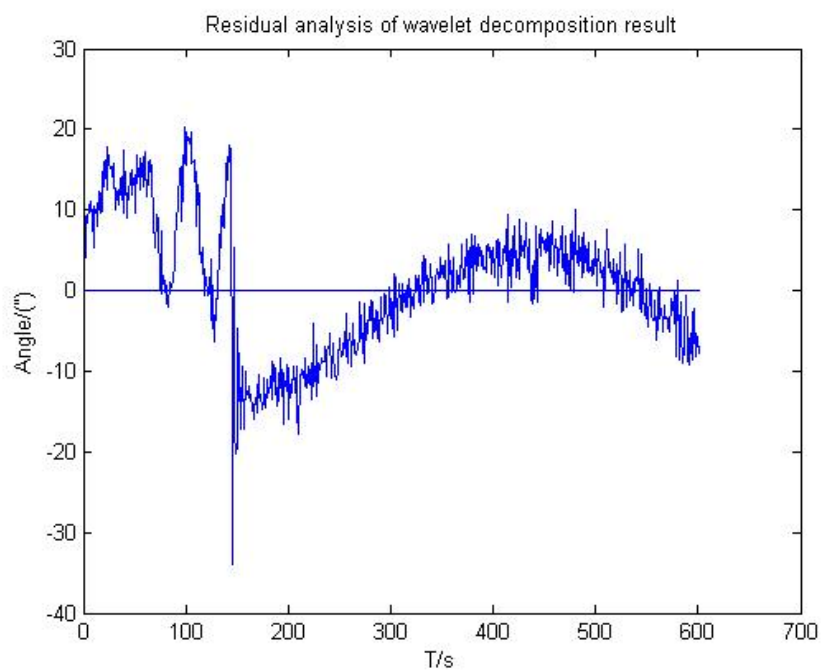



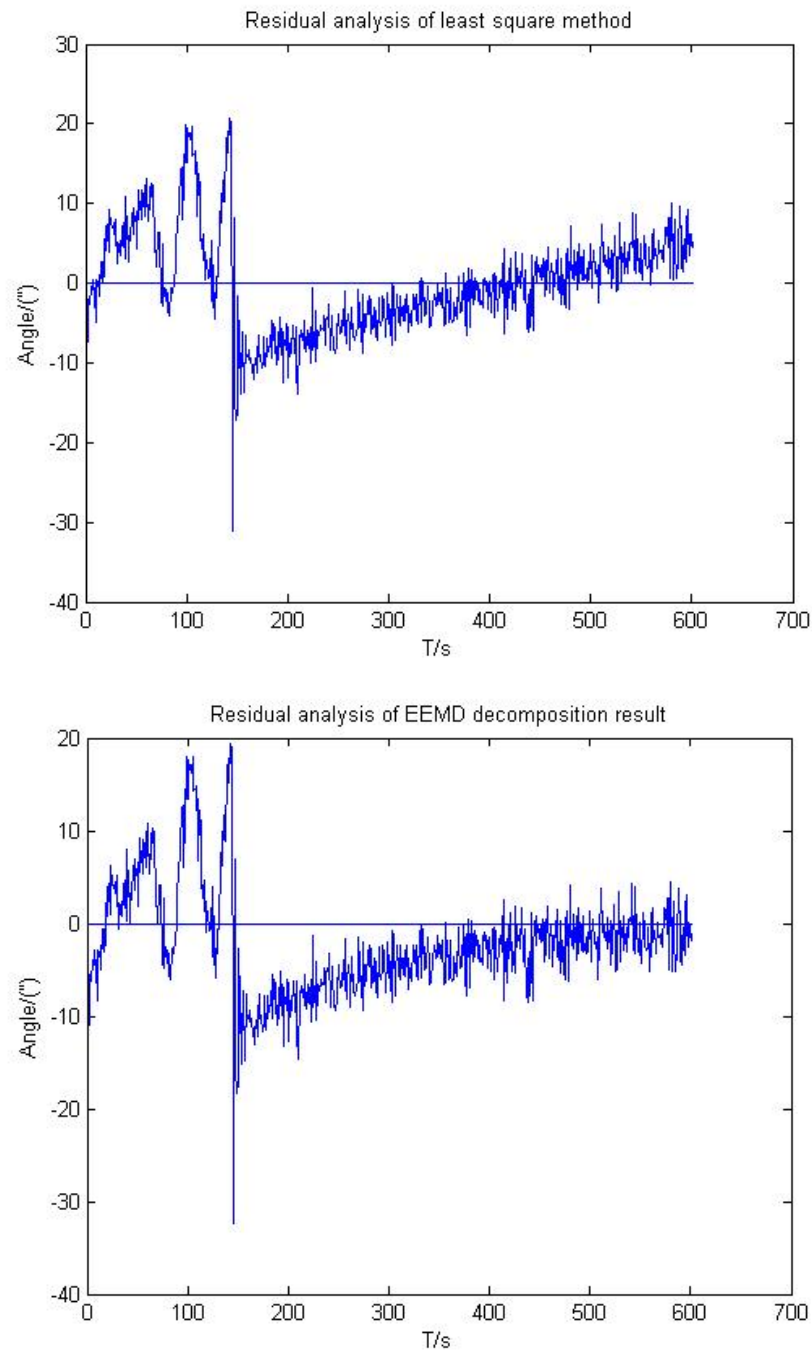

Fig. 2 Residual analysis chart of several filtering methods

The Fig.2 shows that those residues are not parallel to the horizontal axis, and the distance from the horizontal axis vary greatly, so the results of the three methods are Unsatisfactory.

Signal volatility is $b=0.291$, indicating a big amplitude interference, and the wavelet decomposition level should be 8 . Set the low frequency part s8 as input of EEMD, we can get the linear trend term, residual analysis of the result is shown in Fig.3. 

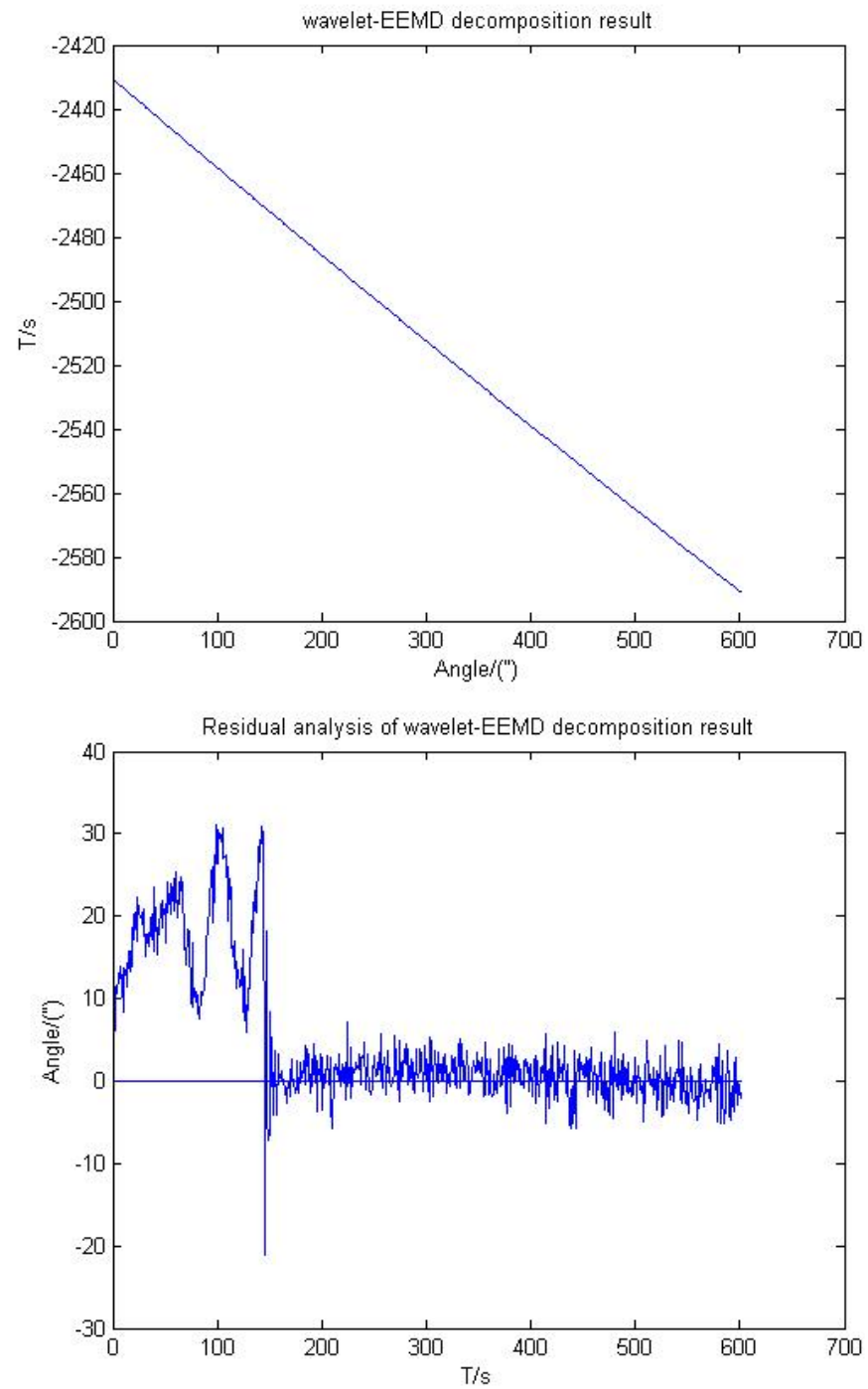

Fig. 3 The result of wavelet-EEMD decomposition and residual analysis Fig.3 shows that the adaptive wavelet-EEMD decomposition algorithm abstracted the platform frame angle signal very well, and the centerline of the residual figure almost parallel to the horizontal axis in addition to points $0-150$, and the points are distributed around the axis 0 , indicating that the magnitude and the slope are accurate.

\section{Conclusion}

In this paper, the adaptive blind source separation algorithm based on wavelet 
- EEMD method abstracted target signal from blind mixture signal successfully and solved the problem of influence of significantly interference effectively. And the introduction of volatility improved the adaptive of wavelet. Simulation test, experiment data validation and some compare are carried on to confirm the proposed method, and the results show that the method has a great advantage, and it can extract the target signals from the disturbed data under significantly interference and greatly improve the alignment accuracy of platform inertial navigation system.

\section{References}

[1]Shuhua Huang. Single channel blind source separation method research and application[D], Taiyuan: Taiyuan University of Science and Technology,2013.

[2] Xinli Wang, Yuanbo Xu. Blind source separation algorithm based on decomposition of the shaker EEMD bearing fault diagnosis [J] Mining Machinery, 2013,4 (41): 85-89.

[3] AYENU-PRAH A Y, ATTOH-OKINE N O. Comparative study of Hilbert-Huang transform, Fourier transform and wavelet transform in pavement profile analysis[S]. Vehicle System Dynami cs, 2009, 47(4): 437A56

[4] Fengshi Chen. Wavelet transform theory and its application in signal processing [M]. National Defense Industry Press,1998.

[5] Norden E Huang, Zheng Shen, Steven R Long. The empirical mode decomposition and the Hilbert spectrum for nonlinear and non-tationary time series analysis [J] .Proc R Soc Lond A, 1998, 454: 903-995

[6] Zhaohua Wu, Huang N E. Advances in Adaptive Data Analysis, 2009, 1 (1): 1. 